\title{
Iatrogenic Aortocoronary Dissection during Routine Coronary Angiography
}

\author{
Santosh Kumar Sinha, Mukesh Jitendra Jha, Vikas Mishra, Puneet Aggarwal \\ Department of Cardiology, LPS Institute of Cardiology, Ganesh Shankar Vidyarthi Memorial Medical College, Kanpur, \\ Uttar Pradesh, India.
}

\section{Corresponding Author:}

Dr. Santosh Kumar Sinha

Email: fionasan@rediffmail.com

This is an Open Access article distributed under the terms of the Creative Commons Attribution License (creativecommons.org/ licenses/by/3.0).

\begin{abstract}
Background: Dissection of coronary artery is rare complication of cardiac catheterization and percutaneous coronary intervention. Its retrograde extension to involve the ascending aorta is even rarer. Case Report: A 62-year-old male, hypertensive, and smoker was admitted with unstable angina. Coronary angiography revealed diffuse subtotal occlusion of proximal left anterior descending artery and tubular lesion with critical stenosis of large obtuse marginal branch. When right coronary artery was cannulated and contrast was injected, there was no regurgitation of contrast from its ostium. Suddenly patient complained of severe chest pain. Contrast spillage was noted from the right coronary artery ostium which revealed huge aorto-ostial dissection extending to ascending aorta. In view of hemodynamic instability, he was urgently transferred for restorative surgery. Conclusion: Aorto-coronary is rare but catastrophic complication of cardiac catheterization which can be successfully managed with surgery.
\end{abstract}

Keywords: Aorta, Chest Pain, Coronary Angiography, Coronary Vessels, Unstable Angina.

\section{Introduction}

Dissection of coronary artery is rare complication of cardiac catheterization and percutaneous coronary intervention. Its retrograde extension to involve the ascending aorta is even rarer. The overall incidence of such dissection is $0.08-0.20 \%$ [1]. It might little higher as more and more complex cases are being performed through percutaneous route because of refinement in technology, improved hardwares and understanding of chronic total occlusion.

\section{Case Report}

A 62-year-old male, hypertensive, and smoker presented with exertional angina, Canadian Cardiovascular Society (CCS) class III angina despite guideline directed medical treatment for past two years. He was admitted with unstable angina. Coronary angiography was performed after proper consent which revealed diffuse subtotal occlusion of proximal left anterior descending artery (LAD), and tubular lesion with critical stenosis of large obtuse marginal branch (OM) [Fig.1]. Right coronary artery (RCA) was cannulated with 6F Judkins Right (JR) diagnostic catheter. Contrast was injected to opacify RCA. On contrast injection, there was no regurgitation of contrast from its ostium which was indicative of fact that tip of catheter was either in the plaque, or the disease was starting right from the ostium [Fig.2a]. Suddenly patient complained of severe chest pain. Contrast spillage was noted from the RCA ostium showing aorto-ostial dissection of RCA [Fig.2b]. Electrocardiogram showed ST elevation in inferior leads (II, III, and aVF). The patient became hemodynamically unstable. Intravenous fluid was started. Further cineview showed huge aorto-ostial dissection extending to ascending aorta [Fig.2c]. As patient had triple vessel disease with large, 


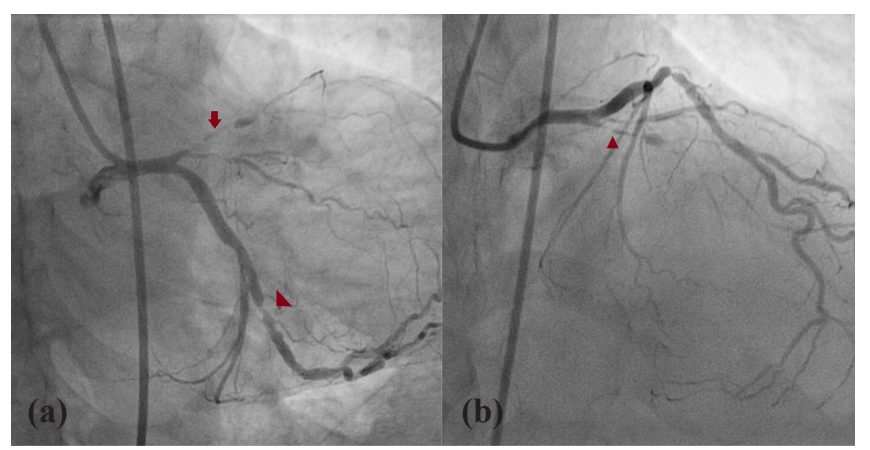

Fig.1: Coronary angiography showing diffuse subtotal occlusion of proximal LAD, and tubular lesion with critical stenosis of large OM; (a) AP caudal view; (b) AP cranial view.

progressive aorto-ostial dissection, he was urgently transferred for restorative surgery.

\section{Discussion}

Aortic involvement in aortocoronary dissection for conventional angiography has been classified according to the Dunning [2]. The level of the most superior extent of the intimal flap marks the highest level of aortic extension where grade 1 dissection flap involves the ipsilateral sinus of Valsalva only; grade 2 flap involves the sinus of Valsalva and the tubular portion of the ascending aorta for a length of $\leq 4 \mathrm{~cm}$; and grade 3 dissection flap extends into the ascending aorta for $\geq 4 \mathrm{~cm}$. The guidelines for treating aortic dissection caused by percutaneous coronary intervention are not there. Surgical interventions are considered if a dissection extend more than $4 \mathrm{~cm}$ into the ascending aorta. In some cases, localized aorto-coronary dissections that appear stable throughout the procedure can probably be managed conservatively [3]. The most important factor to guide treatment is the patient's hemodynamic stability and other associated comorbidities. In our case, it was Dunning grade III dissection. Depending on the background angiogram, and hemodynamic instability, surgery was best option. Surgical interventions in the aorta, by either aortic replacement or glue aortoplasty

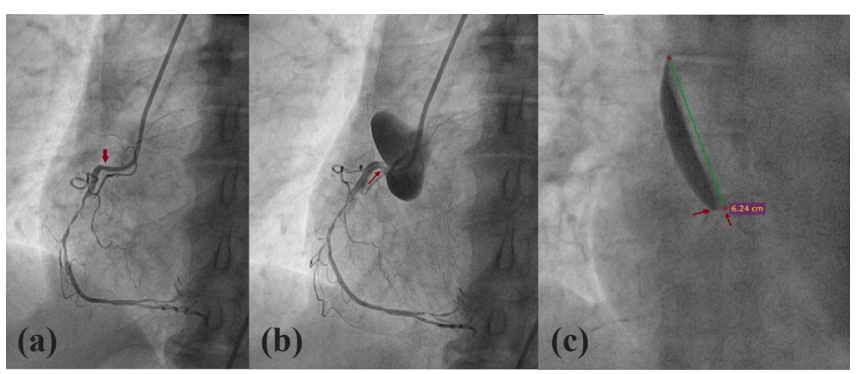

Fig.2:(a) Angiogram of $R C A$ : there was no regurgitation of contrast from its ostium; (b) Contrast spillage was noted from its ostium showing aorto-ostial dissection; (c) Further cine view showed huge aorto-ostial dissection extending to ascending aorta measuring $6.2 \mathrm{~cm}$.

along with aortocoronary bypass, are the various options depending on angiogram [4].

\section{Conclusion}

Aorto-coronary is rare but catastrophic complication of cardiac catheterization. If patient is stable, policy of watchful waiting can be adopted but unstable patients require emergent surgery.

Contributors: SKS: manuscript editing, patient management and conception of the work; MJJ, VM, PA: manuscript writing, literature review and patient management. SKS will act as a study guarantor. All the authors approved the final version of this manuscript and are responsible for all aspect of the study.

Funding: None; Competing interests: None stated.

\section{References}

1. Boyle AJ, Chan M, Dib J, Reasr J. Catheter-induced coronary artery dissection: risk factors, prevention and management. J Invasive Cardiol. 2006;18:500-503.

2. Dunning DW, Kahn JK, Hawkins ET, O'Neill WW. Iatrogenic coronary artery dissections extending into and involving the aortic root. Catheter Cardiovasc Interv. 2000;51:387-393.

3. Hunt I, Faircloth ME, Sinha P, Marber M. Aortocoronary dissection complicating angioplasty of chronically occluded right coronary arteries: is a conservative approach the right approach? J Thorac Cardiovasc Surg. 2006;131:230-231.

4. Ahmed AA, Mahadevan VS, Webb SW, Simon W. Glue aortoplasty repair of aortic dissection after coronary angioplasty. Ann Thorac Surg. 2001;72:922-924. 